\title{
WETTING AND SPREADING OF LIQUID METALS ON SOLID METAL SURFACES
}

$\mathrm{D}^{\mathrm{s}}$ EVELOPMENTS in modorn science which open up new fields of academic and industrial activity also have a direct impact on the older, established applied sciences. These generally undergo a transition from empiricism to the development of the scientific basis of the subject. In most cases this transition has already taken place or is well under way, but it is only just beginning in the heterogeneous group of subjects, soldering, brazing, welding and the gas. cutting of metals, which comprises one aspect of the science of metallurgy. The demand of modern industry to be able to join an ever-increasing variety of metals and alloys by a multitude of techniques has resulted in a considerable expansion of research activity in industry, research associations and the universities. One hundred and forty representatives of these bodies attended a conference on "The Wetting and Spreading of Liquid Metals on Solid Metal Surfaces" held at the University of Birmingham on September 25 under the chairmanship of Prof. E. C. Rollason, head of the Department of Industrial Metallurgy. In his opening address, Prof. Rollason explained that it was planned to hold oocasional conferences on various aspects of the joining of metals, of which this was the first. In this way the practical experience of industrialists could aid the University in deciding the direction of its efforts and further the formation of general principles which are important in the teaching of the undergraduate. In turn, the University hoped to provide a useful stimulus to industry by contributing another point of view. Four papers, by Mr. D. R. Milner, of the Department of Industrial Metallurgy; Mr. E. V. Beatson, of Joseph Lucas, Ltd.; Dr. J. C. Chaston and Mr. M. H. Sloboda, of Johnson Matthey, Ltd. ; and Mr. A. Cibula, of the British Non-Ferrous Metals Research Association, were then presented and discussed.

The phenomena of wetting and spreading are of prime importance in the formation of brazed and soldered joints, and in practice often present con. siderable difficulties, while scientifically they require an interesting extension of classical physical and chemical principles to take account of the complexities and interactions which occur in practical systems. Thus, the well-known equilibrium surface tension theory which relates, first, the surface free energies to the interfacial energy and contact angle; and secondly, the pressure difference across a curved liquid surface to its radii of curvature and surface free energy, implicitly assumes no interaction between the solid and liquid and that the solid surface is plane and clean. In all soldering and brazing systems, however, there is some interaction which cannot be ignored. Mr. Cibula's paper was largely concerned with one of the most important of these, namely, alloying between the liquid and solid metal, which can become of over-riding importance at high tem. peratures. Thus, a liquid drop when placed on the solid surface takes up a shape dependent on the surface and interfacial free energies and then spreads out as alloying takes place, the rate of spread depending on the solubility of the liquid in the solid metal. However, marked alloying results in erosion of the solid metal and a considerable change in the properties of the liquid. Erosion is generally undesirable and may be controlled by using a mixture consisting of a liquid which has limited intersolubility with the solid and a small proportion of a more soluble metal. Increasing the superheat has a marked effect on the rate of spread as it increases the rate of alloying. A particularly important case of this kind occurs when the liquid flows over the solid and interacts to form an intermetallic compound layer at the solid surface greatly reducing its free energy, with the result that the liquid layer becomes unstable and 'dewets' by retracting into an array of isolated drops.

There are other forms of interaction and it has been shown, for example, that the surface tension of solid copper is reduced to less than half its 'clean' value in the presence of lead due to the absorption of lead vapour. When a flux is used, further interactions are possible; metal can be transferred from the liquid metal on to the solid surface by an electro. chemical mechanism; surface energies can be altered by electro-capillarity forces and chemical reactions can take place between the flux and the metal. The latter is made use of in the so-called 'reaction fluxes' for the soldering and brazing of aluminium; these fluxes contain zinc chloride, the zinc being replaced by the aluminium and deposited as a liquid layer on the aluminium surface. Since zine is much less readily oxidized than aluminium, wetting and spreading are promoted.

Oxide films are present on the majority of metals and have to be removed before satisfactory wetting can occur. Fluxes are often used for this purpose, but the exact mechanism by which they act is obscured by the lack of knowledge of the behaviour of molten salts. One thing is becoming clear, however : although fluxes may remove the initial oxide film, oxygen can still diffuse through to reoxidize the underlying metal and retard spreading and flow. An account of the particularly interesting case of the fluxing of stainless steel was given by Dr. Chaston and Mr. Sloboda. In this case the interaction with oxygen appears to be more complex than usual, as in some fluoride-containing fluxes the chromium is preferentially oxidized out of the surface layers, leaving a deposit of iron fluoride, which, having the relatively low surface energy associated with ionic compounds, gives rise to a large contact angle and poor flow of liquid brazing metal.

Mr. Beatson dealt with the two other common methods of removing oxide films, heating in a reducing atmosphere and heating in vacuo. The reduction of oxides by hydrogen or carbon monoxide has a sound thermodynamic basis and it is not difficult to derive the critical value for the dryness of hydrogen or critical carbon dioxide/carbon monoxide ratio above which the oxide cannot be reduced. Below this value, however, the kineties of the system must be considered and no generalizations are as yet possible, other than that above $1,000^{\circ} \mathrm{C}$. thermodynamically favourable reductions appear to take place within a reasonable period of time, while below this temperature an answer can only be given by resort to experiment. Perhaps the major limitation of the 
thermodynamic approach is that at present it can only be applied to pure metals, whereas in practice it is generally necessary to consider alloys, which are sometimes very complex and often contain a proportion of a metal which forms a highly stable oxide. The use of atomic hydrogen for oxide reduction appears to offer interesting potentialities, as thermodynamically it is considerably more favourable. Since dissociation of molecular hydrogen at the metal surface may constitute a major part of the activation energy involved in oxide reduction, the kinetics may also be more favourable. In this connexion there is an interesting process which is finding increasing application to the frequently occurring problem of the construction of fine thermocouples. The thermocouple wires are twisted together and made the cathode of an electrolytic cell in which the electrolyte is 15 per cent aqueous sodium carbonate solution contained in an iron vessel which is the anode. A potential of $80-220$ volts is applied to give a high current density and atomic hydrogen is freely liberated at the cathode, reducing any oxide, while the heat of recombination suffices to fuse the ends of the wires together.

The developing use of vacuum brazing illustrates the advanced techniques necessary in modern metaljoining practice. The use of vacuum or an inert atmosphere, generally argon in Britain and helium in the United States, is essential for the brazing of the highly reactive metals such as titanium, zirconium and molybdenum. The 'Nimonic' alloys, which are so important in the development of the gas turbine, are also frequently brazed in a vacuum. The manner in which a metal surface is freed from oxide by heating in vacuo is open to conjecture and various opinions were expressed at the Conference, such as solution of the oxide into the metal, or vaporization of the oxide film.

The analysis of the flow of the liquid metal into a joint under the influence of capillary forces presents considerable difficulty. A simple model has been set up in which laminar flow is assumed to talke place, but although the size of the capillary passages and the velocity of flow are well within the limits for laminar flow under steady-state conditions, it is doubtful whether this applies to the 'once and for all' filling of a joint. Experiments made on the flow of solder into capillary passages between copper sheet and tinplate give an actual time of flow about twice that predicted theoretically. Mr. Cibula showed that at high temperature, unless special care is taken, the whole analysis becomes meaningless, because temperature gradients enable the liquid to flow all around the relatively hot periphery of a joint, giving a satisfactory-looking fillet but leaving the colder inner portion unfilled. Alternatively, alloying can occur, increasing the melting point of the liquid before it has time to enter far into the capillary.

The points raised during the discussion clearly reflected the preoccupation of those engaged in solving current problems. Thus the difficulty of maintaining the desired purity of hydrogen and predrying techniques when using production heating furnaces received some discussion ; removal of water vapour and oxygen by zirconium or magnesium turnings within the furnace, and the use of the gases boron trifluoride and silicon tetrafluoride were all mentioned. There was considerable discussion of the problem of voids, which may occupy from 5 per cent to as much as 50 per cent of the total area in brazed joints, and in addition to non-uniform heating it was claimed that shrinkage or gas evolution on cooling, and incomplete oxide removal were all significant. The problem of the wetting of stainless steel and the 'Nimonic' alloys was raised by several speakers; the former can be solved by ensuring the use of adequately dry hydrogen and the latter by the use of a vacuum better than one micron. Difficulties associated with the other less widely used metals such as tungsten and molybdenum were also mentioned, but the range of applications and problems which are now confronting workers in this field was perhaps best illustrated by the representative of a firm manufacturing transistors, who stated that when 'brazing' germanium with indium, wetting and spreading were determined by the dislocation density and indices of the crystal face being brazed.

D. R. MILNER

\title{
NUCLEAR ENERGY AND THE GROWTH OF MAN*
}

\author{
By DR. ARTHUR H. COMPTON
}

Distinguished Service Professor of Natural Philosophy, Washington University, St. Louis

$\mathrm{M}^{\mathrm{x}}$ $Y$ prime concern is the urgency that availability of nuclear energy gives to world-wide co-operation in the cause of humanity. It is toward such co-operation that we must now look for the growth of man.

In the programme of 'Atoms for Peace', emphasis has been laid on the usefulness of the energy of the nucleus. This is as it should be. We are using this enorgy in the form of radiation for diagnosis and healing of disoase. We are using isotopes as tools in science and industry. With the important steps already made, we se $\Theta$ electric power from nuclear furnaces ready to meet a great human need for a long time to come.

* Substance of an address during ceremonies marking the presentation of the Atoms for Peace Award (Nature, 179, 698; 1957) to Prof. Niels Bohr at the National Academy of Sciences, Washington, on October 24.
Consider as an example the use of nuclear radiations in the art of healing. Such use began with the discovery of X-rays and radium at about the turn of the century. Estimates have been made of the effectiveness of these rays in diagnosis and therapy. These estimates are necossarily only approximate; but they do show this, that by the use of these rays throughout the world some tens of millions of lives have been saved. 'This number is roughly the same as that of the lives destroyed in all the world's wars fought since their discovery. In this way alone our concern with atoms and nuclei has opened to a great multitude of men and women more complete physical health and life.

But important as it is, here is not our prime point of emphasis when we think of nuclear energy and the growth of man. 